\title{
Hadronic Event Shapes in $B$ Decay
}

\author{
Michael Luke ${ }^{a}$, Martin J. Savage ${ }^{b}$ and Mark B. Wise ${ }^{c}$ \\ a) Department of Physics, University of Toronto, Toronto, Canada M5S 1A7 \\ b) Department of Physics, Carnegie Mellon University, Pittsburgh PA 15213 \\ c) Department of Physics, California Institute of Technology, Pasadena, CA 91125
}

\begin{abstract}
We compute the differential momentum correlation function for hadrons produced in the decay of $B$-mesons. This measure of hadronic event shapes tests the free $b$-quark decay picture for nonleptonic and semileptonic decays of $B$-mesons. Our results can also be applied to $B_{s}$ and $\Lambda_{b}$ decay.
\end{abstract}

UTPT 93-28

CMU-HEP 93-24

CALT-68-1905

DOE-ER/40682-49

November 1993 
Free heavy quark decay is expected to describe accurately the inclusive weak decays of hadrons containing a heavy quark. There are two physical reasons for this. First, while the individual exclusive modes depend on the details of hadronization, inclusive quantities which sum over all possible decay modes are expected to be insensitive to these details, since for heavy quarks there are many states available to the decay products. Second, for large heavy quark mass $m_{Q}$, the heavy quark in the hadron is almost on-shell and moving with the same four-velocity as the hadron. It is therefore reasonable to treat its decay as that of a free quark.

For semileptonic (and radiative) decays, this duality between hadrons and free quarks and gluons has been shown to follow from QCD as the leading term in an expansion in $1 / m_{Q}$ [1]. At leading order the differential decay rate for inclusive $B \rightarrow X e \bar{\nu}_{e}$ decay, $d \Gamma / d q^{2} d E_{e}\left(q=p_{e}+p_{\nu}\right)$, suitably averaged over $E_{e}$, is equal to that for free $b$-quark decay. Non-perturbative corrections, parameterising the effects of the strong interactions of the heavy $b$-quark with the light degrees of freedom in the $B$-meson, first arise at order $1 / m_{b}^{2}$. These have recently been calculated [2]-[7]. Duality in this instance arises because the current-current correlator for the weak hadronic current is analytic everywhere in the complex energy plane, except at points on the real axis corresponding to physical intermediate states. This allows the phase space integral over the energy of the final hadronic state to be deformed to lie far from the the singularities in the decay amplitude. Along the deformed contour perturbative QCD provides a valid description of the decay products free of infrared singularities. The free quark decay picture emerges at leading order in $1 / m_{b}$.

For nonleptonic decays the above argument does not apply, since there is no longer a kinematic variable that can be used to define a path of integration to be deformed. Nevertheless, the free quark decay picture is physically reasonable and widely used (e.g., to extract $\left|V_{c b}\right|$ from the $B$-meson lifetime). Even if the free quark decay picture holds when $m_{b}$ is very large for the physical value of the $b$-quark mass there is only about $m_{b}-m_{c} \simeq 3 \mathrm{GeV}$ of energy (beyond the charm quark mass) available to the final state particles in weak $B$ decay. Furthermore, this energy is shared amongst three quarks in $b \rightarrow c \bar{u} d$ nonleptonic decay so that the energy that each quark carries is not very large compared with the QCD scale. It is important to test the free quark decay picture in as many ways as possible. One test is provided by the predicted equality of the $B^{0}, B^{-}, B_{s}$ and $\Lambda_{b}$ lifetimes and another by the predicted semileptonic branching ratios. In this letter, we point out that event shapes, characterized by the momentum angular correlation 
distribution, may also be used to test its validity. We calculate this correlation function for both nonleptonic and semileptonic $b$ decays assuming that the free quark decay picture is valid.

Consider the weak decay of a hadron containing a $b$-quark. We define the following quantity, in analogy with the differential energy-energy correlation function used to describe hadronic events at colliders [8]:

$$
\begin{aligned}
\frac{d \Sigma}{d \cos \theta}= & \frac{1}{\Gamma}\left(\sum_{i, j} \int d E_{i} d E_{j} \frac{\left|\vec{p}_{i}\right|}{m_{b}} \frac{\left|\vec{p}_{j}\right|}{m_{b}} \frac{d^{3} \Gamma}{d E_{i} d E_{j} d \cos \theta_{i j}}\right. \\
& \left.+\sum_{i} \int d E_{i} \frac{\left|\vec{p}_{i}\right|^{2}}{m_{b}^{2}} \frac{d \Gamma}{d E_{i}} \delta(1-\cos \theta)\right) .
\end{aligned}
$$

$\Gamma$ is the total nonleptonic or semileptonic decay width, $m_{b}$ is the mass of the initial heavy $b$-quark, and the double sum runs over all pairs of strongly interacting particles $(i, j), i \neq j$, in the final state separated by an angle $\theta_{i j}$, treating $(i, j)$ and $(j, i)$ as distinct pairs. As we have defined it, the correlation function (1) is insensitive to the emission of soft and collinear gluons by the partons in the final state, which is required for it to be free of infrared divergences. Consequently $d \Sigma / d \cos \theta$ at the hadron level should be equal (up to corrections of order $\left.1 / m_{b}\right)$ to its value calculated from free $b$-quark decay. For $b \rightarrow c$ transitions the final state contains a charmed hadron which decays weakly. It is important in the evaluation of (1) that this charmed hadron be treated as a single particle. One should not evaluate the sums in (1) using the charmed hadron's decay products. This complication makes $d \Sigma / d \cos \theta$ more difficult to measure.

For nonleptonic $b$ decay, at leading order in $\alpha_{s}$, the tree level $b \rightarrow q \bar{u} d$ matrix element 
of the weak Hamiltonian gives

$$
\begin{aligned}
& \frac{d^{3} \Gamma}{d y_{q} d y_{d} d \cos \theta_{q d}}=\frac{3 G_{F}^{2} m_{b}^{5}}{2 \pi^{3}}\left(\frac{\left|\hat{p}_{q}\right| y_{d}^{2}\left(y_{q}-\left|\hat{p}_{q}\right| \cos \theta_{q d}\right)\left(1-y_{q}-y_{d}\right)}{1-y_{q}+\left|\hat{p}_{q}\right| \cos \theta_{q d}}\right) \\
& \times \delta\left(y_{d}-\frac{1-2 y_{q}+\hat{m}_{q}^{2}}{2\left(1-y_{q}+\left|\hat{p}_{q}\right| \cos \theta_{q d}\right)}\right) \\
& \frac{d^{3} \Gamma}{d y_{q} d y_{\bar{u}} d \cos \theta_{q \bar{u}}}=\frac{3 G_{F}^{2} m_{b}^{5}}{2 \pi^{3}}\left(\frac{\left|\hat{p}_{q}\right| y_{\bar{u}^{2}}\left(y_{q}-\hat{m}_{q}^{2}-y_{\bar{u}} y_{q}+y_{\bar{u}}\left|\hat{p}_{q}\right| \cos \theta_{q \bar{u}}\right)}{1-y_{q}+\left|\hat{p}_{q}\right| \cos \theta_{q \bar{u}}}\right) \\
& \times \delta\left(y_{\bar{u}}-\frac{1-2 y_{q}+\hat{m}_{q}^{2}}{2\left(1-y_{q}+\left|\hat{p}_{q}\right| \cos \theta_{q \bar{u}}\right)}\right) \\
& \frac{d^{3} \Gamma}{d y_{\bar{u}} d y_{d} d \cos \theta_{\bar{u} d}}=\frac{3 G_{F}^{2} m_{b}^{5}}{2 \pi^{3}}\left(\frac{y_{\bar{u}^{2}} y_{d}^{2}\left(1-y_{\bar{u}}+y_{\bar{u}} \cos \theta_{\bar{u} d}\right)}{1-y_{\bar{u}}+y_{\bar{u}} \cos \theta_{\bar{u} d}}\right) \\
& \times \delta\left(y_{d}-\frac{1-2 y_{\bar{u}}-\hat{m}_{q}^{2}}{2\left(1-y_{\bar{u}}+y_{\bar{u}} \cos \theta_{\bar{u} d}\right)}\right)
\end{aligned}
$$

where $y_{q}=E_{q} / m_{b}, y_{\bar{u}}=E_{u} / m_{b}, y_{d}=E_{d} / m_{b}, \hat{p}_{q}=p_{q} / m_{b}$ and $\hat{m}_{q}=m_{q} / m_{b}$. For the case, $q=u$, the energy integral in (1) may be performed analytically, and the correlation function is found to be

$$
\begin{aligned}
\left.\frac{d \Sigma}{d \cos \theta}\right|_{m_{q=0}=} & \frac{8}{(1-\cos \theta)^{7}}\left[(1-\cos \theta)\left(811+1163 \cos \theta+401 \cos ^{2} \theta+25 \cos ^{3} \theta\right)\right. \\
& \left.+6(1+\cos \theta)\left(195+171 \cos \theta+33 \cos ^{2} \theta+\cos ^{3} \theta\right) \log \frac{1+\cos \theta}{2}\right] \\
& +\frac{11}{30} \delta(1-\cos \theta) .
\end{aligned}
$$

When $q=c$ the mass of the charm quark cannot be neglected and, the phase space integration must be done numerically. In fig. 1 the momentum correlation functions for $q=u$ (solid curve) and $q=c$ (dashed curve) are shown for $m_{b}=4.8 \mathrm{GeV}$ and $m_{c}=$ $1.6 \mathrm{GeV}$. In both cases, the correlation function is strongly peaked at $\theta=\pi$, along with the delta function at $\theta=0$. In addition to corrections suppressed by powers of $1 / m_{b}$, there are computable $\mathcal{O}\left(\alpha_{s}\left(m_{b}\right)\right)$ corrections to the results in fig. 11.

For comparison with experiment, it is also useful to define the Fox-Wolfram moments $H(L)$ [9] of the distribution (1):

$$
H(L)=\int d \cos \theta \frac{d \Sigma}{d \cos \theta} P_{L}(\cos \theta)
$$


where $P_{L}(x)$ are Legendre polynomials. The first few Fox-Wolfram moments $H(L)-H(0)$ (we have subtracted $H(0)$ to remove the effect of the delta function at $\theta=0$ ) are presented in Table 1. $H(1)$ is equal to zero because of momentum conservation. Conservation of energy implies, in the massless $q=u$ case, that $H(0)=1$.

We now consider inclusive semileptonic $B$ decays. At leading order in $\alpha_{s}$ there is only one quark in the final state, so the correlation function is proportional to a delta function at $\theta=0$ :

$$
\begin{gathered}
\frac{d \Sigma^{(0)}}{d \cos \theta}=\frac{2}{15} \frac{1-9 \hat{m}_{q}^{2}+45 \hat{m}_{q}^{4}-45 \hat{m}_{q}^{8}+9 \hat{m}_{q}^{10}-\hat{m}_{q}^{12}+120 \hat{m}_{q}^{6} \log \hat{m}_{q}}{1-8 \hat{m}_{q}^{2}+8 \hat{m}_{q}^{6}-\hat{m}_{q}^{8}-24 \hat{m}_{q}^{4} \log \hat{m}_{q}} \\
\times \delta(1-\cos \theta) .
\end{gathered}
$$

The leading perturbative contribution to the semileptonic correlation function away from $\cos \theta=1$ arises from gluon bremsstrahlung at $\mathcal{O}\left(\alpha_{s}\right)$, and may be calculated using the results of [10]. The rate for the process $b \rightarrow q g e \bar{\nu}_{e}$ is

$$
\Gamma=\frac{1}{2 m_{b}} \frac{1}{(2 \pi)^{8}} \int \frac{d^{3} p_{c}}{2 E_{c}} \frac{d^{3} k}{2 E_{g}} \frac{d^{3} p_{e}}{2 E_{e}} \frac{d^{3} p_{\nu}}{2 E_{\nu}} \delta^{4}\left(p_{b}-p_{c}-p_{e}-p_{\nu}-k\right) \frac{1}{2}|\mathcal{M}|^{2},
$$

where the spin averaged matrix element for is

$$
\begin{aligned}
& \frac{1}{2}|\mathcal{M}|^{2}=G_{F}^{2} g_{s}^{2} \operatorname{Tr}\left[\not p_{e} \gamma_{\mu} \not p_{\nu} \gamma_{\beta}\left(1-\gamma_{5}\right)\right] . \\
& \operatorname{Tr}\left[\not p_{c}\left(\frac{2 p_{c}^{\lambda}+\gamma^{\lambda} \not k}{p_{c} \cdot k} \gamma_{\mu}-\gamma_{\mu} \frac{2 p_{b}^{\lambda}-\not k \gamma^{\lambda}}{p_{b} \cdot k}\right) \not p_{b}\left(\gamma_{\beta} \frac{2 p_{c \lambda}-\not k \gamma_{\lambda}}{p_{c} \cdot k}-\frac{2 p_{b \lambda}-\gamma_{\lambda} \not k}{p_{b} \cdot k} \gamma_{\beta}\right)\right],
\end{aligned}
$$

and $k$ is the gluon four-momentum. The integration of the lepton phase space gives

$$
\int \frac{d^{3} p_{e}}{2 E_{e}} \frac{d^{3} p_{\nu}}{2 E_{\nu}} \operatorname{Tr}\left[\not p_{e} \gamma_{\mu} p_{\nu} \gamma_{\beta}\left(1-\gamma_{5}\right)\right]=\frac{2 \pi}{3}\left[P_{\mu} P_{\beta}-g_{\mu \beta} P^{2}\right]
$$

where $P=p_{e}+p_{\nu}$. Once again, the integration can be performed analytically when the final quark is massless, and the $\mathcal{O}\left(\alpha_{s}\right)$ contribution to the correlation function is found to be

$$
\begin{aligned}
& \left.\frac{d \Sigma^{(1)}}{d \cos \theta}\right|_{m_{q=0}}=-\frac{4 \alpha_{s}}{3 \pi}\left(\frac{(83-5 \cos \theta)(1+\cos \theta)^{3}}{3(1-\cos \theta)^{7}} \log \frac{1+\cos \theta}{2}\right. \\
& \left.+\frac{6875+17373 \cos \theta+12168 \cos ^{2} \theta+1468 \cos ^{3} \theta-507 \cos ^{4} \theta+63 \cos ^{5} \theta}{360(1-\cos \theta)^{6}}\right)
\end{aligned}
$$

where we have neglected the $\mathcal{O}\left(\alpha_{s}\right)$ contribution to the delta function. The contribution of $d \Sigma^{(1)} / d \cos \theta$, in eq. (9), to $H(0)$ diverges. This divergence is cancelled by contribution of 
the order $\alpha_{s}$ part of the delta function (that we neglected) to $H(0)$. For a massive $c$ quark in the final state, we have performed the phase space integration numerically. The results are plotted for $q=u$ and $q=c$ in fig. 2. Unfortunately, for both $q=u$ and $q=c$ there is only a small contribution to the correlation function away from the forward direction, making these distributions extremely difficult to measure.

The first few Fox-Wolfram moments $H(L)-H(0)$ for semileptonic $b$ decays are presented in Table 2. Note that these moments are much larger for $b \rightarrow u$ transitions than $b \rightarrow c$ transitions. The large charm quark mass suppresses the amplitude for gluon bremsstrahlung.

Clearly the results given in this paper also hold for $\Lambda_{b}$ and $B_{s}$ decay. For the $q=u$ case, where the final quark is massless, our results can be applied to charm decay. However, the difference in lifetimes between the $D^{+}$and $D^{0}$ is a clear sign that the free quark decay picture breaks down badly for nonleptonic charm decays, and so we do not expect our results to be useful in that case.

Finally, we note that if the predictions for $b$ decay event shapes that we have made disagree with experiment, it will not provide conclusive evidence that the free quark decay picture fails for the total nonleptonic decay width. It is possible that corrections suppressed by powers of $1 / m_{b}$ are larger for event shapes than for the nonleptonic width.

We thank H.D. Politzer and L. Wolfenstein for useful discussions. This research was supported in part by the Department of Energy under contract DE-FG02-91ER40682 and DE-FG03-92-ER40701. 


\section{References}

[1] J. Chay, H. Georgi and B. Grinstein, Phys. Lett. 247B (1990) 399.

[2] I.I. Bigi, N. G. Uraltsev and A. I. Vainshtein, Phys. Lett. $293 B$ (1992) 430.

[3] I. I. Bigi, B. Blok, M. Shifman, N. G. Uraltsev and A. Vainshtein, TPI-MINN-92/67-T (1992); Phys. Rev. Lett. 71 (1993) 496.

[4] B. Blok, L. Koyrakh, M. Shifman and A. Vainshtein, NSF-ITP-93-68 (1993), hepph/9307247.

[5] A. V. Manohar and M. B. Wise, UCSD-PTH 93-14 (1993), hep-ph/9308246.

[6] T. Mannel, IKDA 93/16 (1993), hep-ph/9308262.

[7] A. F. Falk, M. Luke and M. J. Savage, UCSD-PTH 93-23 (1993), hep-ph/9308288.

[8] V. Barger and R. Phillips, Collider Physics, Addison-Wesley (1987) p. 291.

[9] G. Fox and S. Wolfram, Phys. Rev. Lett. 23 (1979) 1581

[10] B.Guberina, R.D. Peccei and R. Ruckl, Nucl. Phys. B171 (1980) 333. 


\section{Figure Captions}

Fig. 1. The momentum correlation function $d \Sigma / d \cos \theta$ for nonleptonic decays computed in the parton model to lowest order in $\alpha_{s}$. The solid curve corresponds to the case when all three quarks in the final state are massless. The dashed curve corresponds to the case for a $b$ quark decaying to a charmed final state.

Fig. 2. The momentum correlation function for (a) $b \rightarrow u g e \bar{\nu}_{e}$ and (b) $b \rightarrow c g e \bar{\nu}_{e}$ as a function of $\cos \theta$, omitting the normalization factor $4 \alpha_{s} / 3 \pi$. The dominant contribution to the delta function at $\theta=0$ comes from the tree-level graph; away from $\theta=0$ the distribution arises from the parton-level decay $b \rightarrow q g e \bar{\nu}_{e}$. 


\begin{tabular}{|c|c|c|}
\hline & \multicolumn{2}{|c|}{$H(L)-H(0)$} \\
\hline$L$ & $m_{q}=0$ & $m_{q}=1.6 \mathrm{GeV}$ \\
\hline 1 & -1.0 & -0.72 \\
2 & -0.44 & -0.31 \\
3 & -0.74 & -0.54 \\
4 & -0.57 & -0.41 \\
5 & -0.67 & -0.49 \\
6 & -0.61 & -0.44 \\
\hline
\end{tabular}

Table 1: Fox-Wolfram Moments for Nonleptonic $B$ Decay

\begin{tabular}{|c|c|c|}
\hline & $3 \pi / 4 \alpha_{s}\left(m_{b}\right) \times(H(L)-H(0))$ \\
\hline$L$ & $m_{q}=0$ & $m_{q}=1.6 \mathrm{GeV}$ \\
\hline 1 & -0.15 & -0.028 \\
2 & -0.26 & -0.037 \\
3 & -0.32 & -0.035 \\
4 & -0.36 & -0.035 \\
5 & -0.40 & -0.034 \\
6 & -0.43 & -0.034 \\
\hline
\end{tabular}

Table 2: Fox-Wolfram Moments for Semileptonic $B$ Decay 


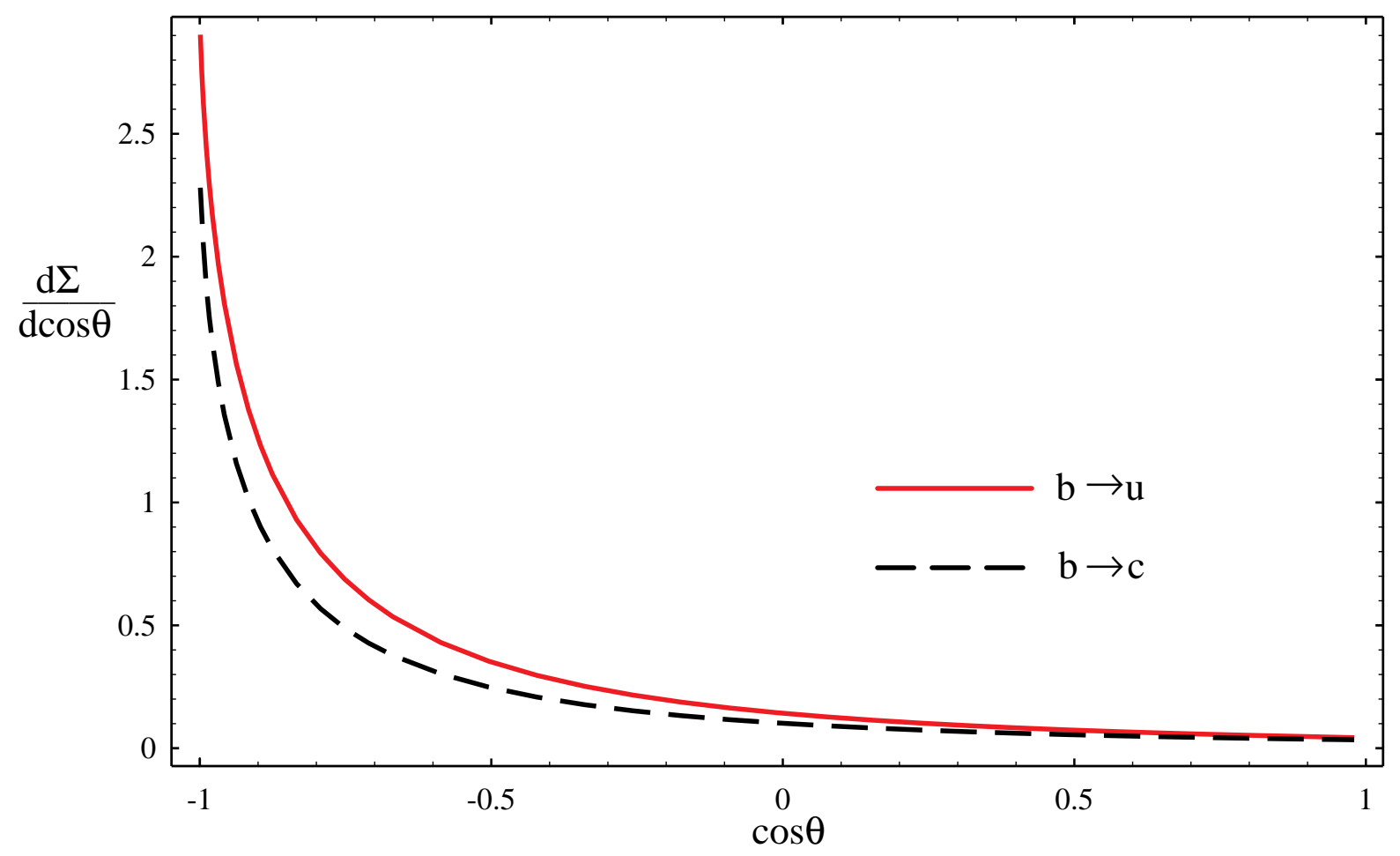

Figure 1

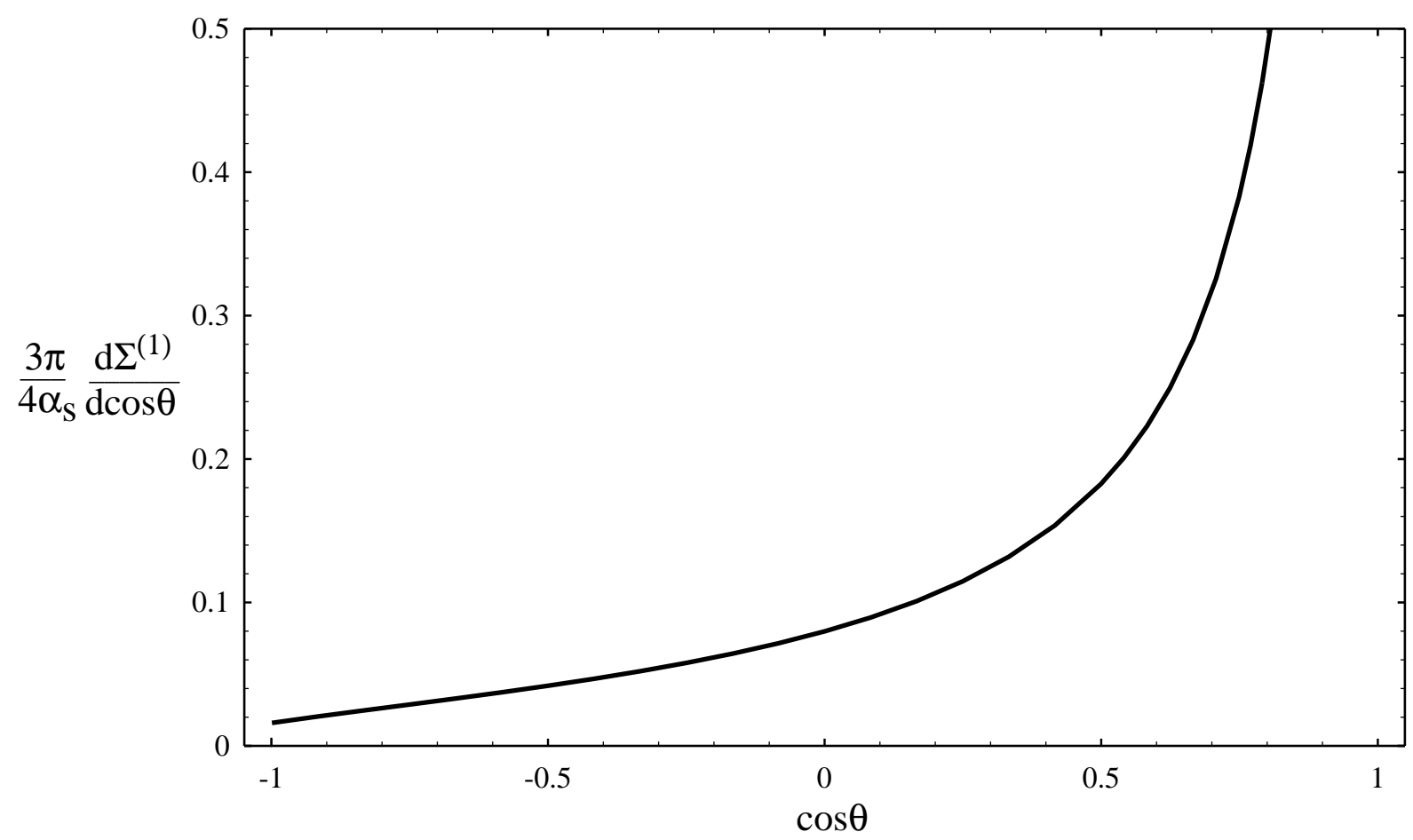

Figure 2 (a) 


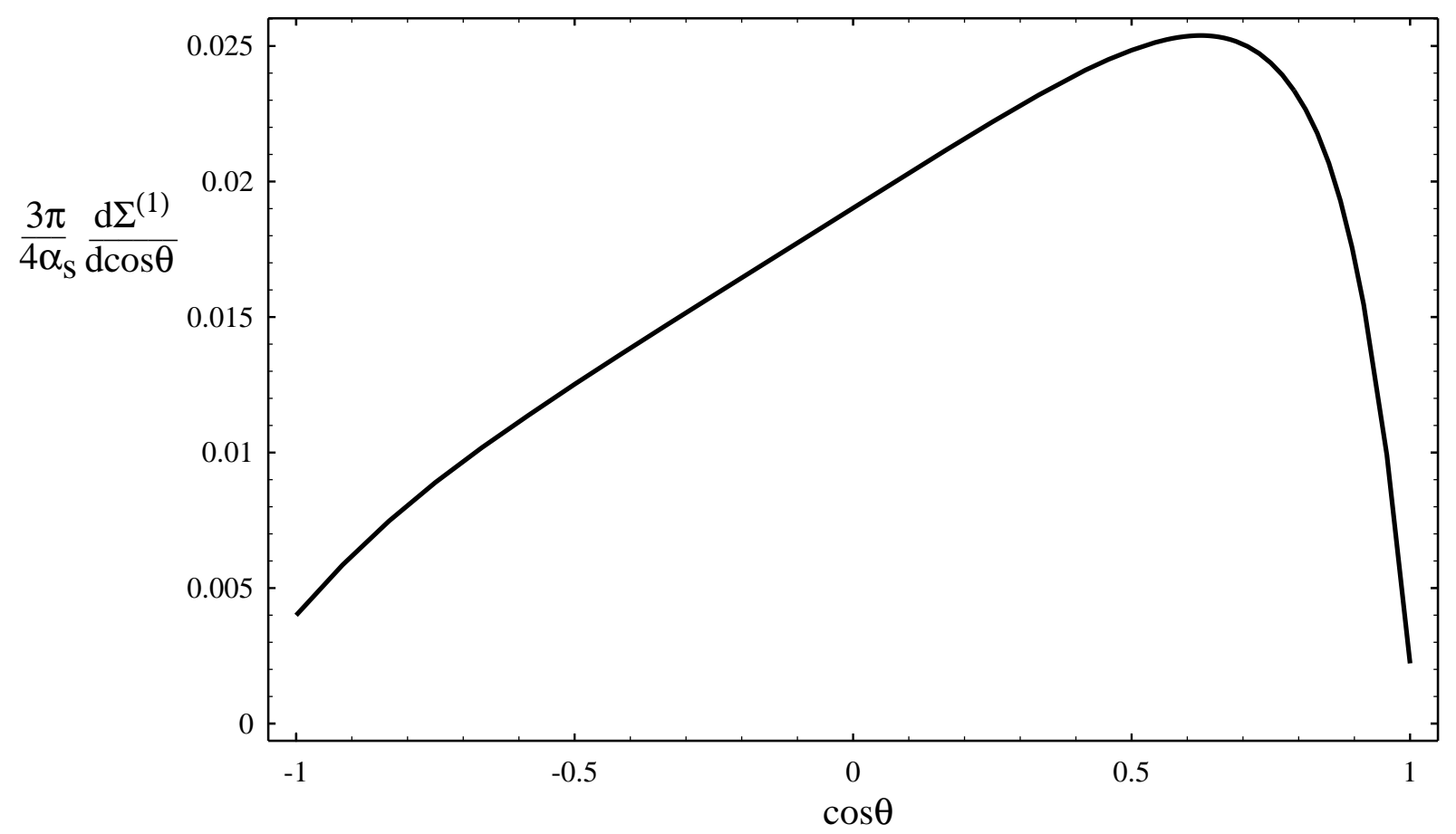

Figure 2 (b) 
This figure "fig1-1.png" is available in "png" format from: http://arxiv.org/ps/hep-ph/9311308v1 
This figure "fig1-2.png" is available in "png" format from: http://arxiv.org/ps/hep-ph/9311308v1 
This figure "fig1-3.png" is available in "png" format from: http://arxiv.org/ps/hep-ph/9311308v1 